Dedicated to the memory of Glyn Humphreys.

\title{
Retinotopic information interacts with category selectivity in human ventral cortex
}

\author{
Fatma Uyar ${ }^{1}$, Sarah Shomstein ${ }^{1}$, \\ Adam S. Greenberg ${ }^{2}$ and Marlene Behrmann ${ }^{2}$
}

\footnotetext{
${ }^{1}$ Department of Psychology, George Washington University, Washington, DC 20052

${ }^{2}$ Department of Psychology and the Center for the Neural Basis of Cognition, Carnegie Mellon University, Pittsburgh PA
}

Keywords: retinotopy, category selectivity, face processing, fMRI, scene processing, functional connectivity

Correspondence should be addressed to:

Marlene Behrmann, Department of Psychology, Carnegie Mellon University, Pittsburgh PA 152133890, USA. Email: behrmann@cmu.edu 


\begin{abstract}
Until recently, the general consensus with respect to the organization of ventral visual cortex is that early, retinotopic regions are sensitive to the spatial position of the input stimuli whereas later, higher-order regions are sensitive to the category of the input stimuli. Growing recognition of the bidirectional connectivity of the visual system has challenged this view and recent empirical evidence suggests a more interactive and graded system. Here, based on findings from functional MRI in adult observers, in which meridians and category selective regions are localized and their activation sampled, we support this latter perspective by showing that category effects are present in retinotopic cortical areas and spatial position effects are present in higher-order regions. Furthermore, the results indicate that the retinotopic and later areas are functionally connected suggesting a possible mechanism by which these seemingly disparate effects come to be intermixed in both early and later regions of the visual system.
\end{abstract}




\section{Introduction}

Until fairly recently, there has been a general consensus that more posterior, earlier, parts of the visual cortical hierarchy are governed by principles of topography, retaining an isomorphic relationship between the location of the stimulus in the world and the activation of particular regions of early visual cortex. In contrast, the more anterior, later, cortical regions are tuned to respond to particular categories of objects (for example, faces or houses), abstracted away from spatial location of the input stimuli. These two principles, 'spatial specificity' and 'category specificity', are thought to guide the transition from positional specificity instantiated in the progression from small to larger receptive field sizes as one moves caudally to rostrally in the visual cortex to position independence and the ability to generalize across higher-order changes, including viewing angle, pose and size (Robert Desimone \& Gross, 1979; Kobatake \& Tanaka, 1994). Thus, the standard view has been that the goal of the computation of the ventral visual cortex is to derive stable and invariant properties of the perceptual input by gradually abstracting away low-level properties of the input through the derivation of more conceptual representations. Similar arguments have been made with respect to the dorsal system, as well (Roth \& Zohary, 2015).

There has, however, been growing recognition that this strict division into retinotopic versus non-retinotopic cortex may not hold in this binary fashion. Accumulating evidence has led to an alterative conceptualization of ventral visual cortex which posits that, in a fully connected bidirectional network (Felleman \& Van Essen, 1991), even more anterior, higher-order regions, standardly associated with the representation of more complex patterns, might reflect some of the topographic constraints of the area from which signals are received. And, by the same token, the activation profile of early visual areas which are considered primarily driven by and responsive to the input topography, perhaps on a purely feedforward basis, might reflect some of the 
properties of higher-order (even category-selective) areas perhaps by virtue of receiving feedback signals from these more anterior regions (for recent discussion of this point, see (Furl, 2015)).

Support for the idea of an interactive and more graded system that results in a mixture of positional and category specificity in the visual system has been gleaned from various studies (for comprehensive review, see (Kravitz, Saleem, Baker, Ungerleider, \& Mishkin, 2013)). As we review below, some of these investigations have argued for positional and retinotopic influences in later parts of the visual system and some have provided evidence of category-selective effects in earlier spatially-organized parts of the visual system. To our knowledge, however, there has been no consideration of the functional connectivity between earlier and later parts of the visual system that would permit and facilitate the bidirectional influence of retinotopic properties and category specificity. The focus of this manuscript, then, is, first, to characterize the influence of positional (as well as category) specificity in high-level parts of the visual system and the influence of category (as well as positional) information in early parts of the visual system. Thereafter, we explore the functional connectivity between the low-level and high-level regions so as to elucidate the bidirectional influence of these principles.

\subsection{Retinotopic effects in higher-level cortical regions}

One way of exploring the influence of the spatial position of the stimulus is to examine whether the known low-level preference for contralateral over ipsilateral stimuli is also observed in highlevel visual areas. Unsurprisingly, in one study aimed to explore this, participants who viewed faces, objects, scenes and scrambled images shown in the right or left visual fields evoked greater BOLD signal in primary visual cortex for contralateral stimuli. Of note, although this contralateral preference was greater in low-level regions, all regions examined (including Lateral Occipital (LO), Occipital Face Area (OFA), posterior fusiform and Fusiform Face Area (FFA)), revealed this 
preference, indicating that sensitivity to stimulus position is evident even in high-level ventral cortex (Hemond, Kanwisher, \& Op de Beeck, 2007). This finding has now been replicated several times (McKyton \& Zohary, 2007; Niemeier, Goltz, Kuchinad, Tweed, \& Vilis, 2005), and there are now many reports demonstrating location representations in anterior ventral areas (Arcaro, McMains, Singer, \& Kastner, 2009; Kravitz, Kriegeskorte, \& Baker, 2010; Schwarzlose, Swisher, Dang, \& Kanwisher, 2008; Strother, Aldcroft, Lavell, \& Vilis, 2010). Interestingly, detailed scrutiny of positional and category specificity reveals their co-existence (and potentially even equal strength) in some high-level regions. Using fMRI and MVPA analyses exploring correlations between even and odd runs in LOC (Lateral Occipital Complex), FFA, EBA (Extrastriate Body Area) and PPA (Parahippocampal Place Area), Golomb and Kanwisher (2012) have shown that the highest correlations in all these regions were for the same category of image presented in the same combined location. The rank ordering of the remaining categories were as predicted, with weaker correlations when the stimuli were from the same category but in different locations or when location was preserved but category differed. The weakest correlation was observed for those comparisons when both category and location differed.

A similar pattern of the co-existence of position and category specificity has also been obtained in neural recording in non-human primates. For example, using high-resolution fMRI, Rajimehr et al. (2014) showed that distinct subregions within face-selective patches showed a coarse retinotopic map of eccentricity as well as of polar angle. Other patches revealed a retinotopic bias just to a specific location of the visual field and yet others showed no retinotopic selectivity. Retinotopic selectivity has even been documented in the anterior inferotemporal cortex (AIT) in a study in which both behavioral and neural responses were recorded in response to visual forms whose retinal position was varied. Unsurprisingly, AIT neurons were highly selective for the forms. The counterintuitive, and more relevant, result for the current purpose was the 
sensitivity to retinal position with an approximately $60 \%$ response decrease between positions within $+/-1.5$ degrees of the center of gaze (DiCarlo \& Maunsell, 2003). In a related investigation using a large set of naturalistic visual images containing a range of real world objects that varied along object position, size, and pose variables, recordings obtained in IT and V4 also revealed that information about these so-called 'low-level variables' is explicitly coded in both V4 and IT, albeit to a lesser extent in the latter than former (Hong, Yamins, Majaj, \& DiCarlo, 2016). Moreover, a convolutional neural network optimized for performance on a categorization task, accounted well for their empirical data, leading to the conclusion that the role of pooling in the ventral stream is not to discount object transformations as one progresses to more anterior regions, but, rather, to preserve some of this information.

Last, Hung et al. (2005) used a biologically plausible, classifier-based readout technique to investigate the neural coding of selectivity and invariance at the IT population level and revealed robust information about both object "identity" and "category" that was invariant over a range of object positions and scales in IT. Of relevance here, as in Hong et al. (2016), coarse information about position and scale could be read out from the very same neuronal population.

\subsection{Category-specific effects in earlier visual cortex}

As is clear from the evidence cited above, many studies, using a host of different methods, provide support for the view that later, higher-order regions of the ventral visual cortex retain information about low-level stimulus dimensions (location, size, pose). In complementary fashion, there are some (although admittedly, fewer) studies showing that early areas of visual cortex demonstrate some category-selectivity. One possible reason for the relative paucity of evidence is that category differences that manifest in retinotopic areas may result from differences in fundamental stimulus properties (for example, houses may contain more high-frequency information than faces) or any 
of a host of other image-based differences. Under controlled conditions, separability of category information is generally not observed in V1 or V2 (Grill-Spector \& Weiner, 2014) although it has been shown that PPA showed a significantly greater response magnitude to the upper versus lower field images compared to the lower field images. In contrast, the FFA, EBA and LO exhibited opposite effects and greater response magnitudes to the lower field images compared with the upper field images (Sayres \& Grill-Spector, 2008; Schwarzlose, et al., 2008).

One relevant finding, however, that provides some evidence for category-selectivity in early visual cortex comes from a recent investigation using intracranial recording in children. This study demonstrated preferential neural activation to an upright versus inverted face, as reflected by roughly $23 \%$ augmentation of high-gamma activity at $80-150 \mathrm{~Hz}$ in lower-order visual cortex, roughly corresponding to V1 and V2, within the first 100 ms post-stimulus presentation (Matsuzaki, Schwarzlose, Nishida, Ofen, \& Asano, 2015). This very early activation profile is compatible with findings from magnetoencephalography (MEG) showing that face and house stimuli activate $\mathrm{V} 1$ around $40 \mathrm{~ms}$ post stimulus onset, with the amplitude elicited by face stimuli significantly larger than that elicited by house stimuli (Shigihara \& Zeki, 2014). Although in some cases, this apparent category difference may be a function of differences in some low-level properties of the stimuli, the amplitude enhancement for faces may also reflect some categoryselectivity as in the case of upright versus inverted faces (where low-level image properties are matched). Moreover, the category-selectivity might reflect tuning of neurons as a function of retinal position at which faces and houses are typically observed in daily life and thus be a product of real-world statistics and experience (Levy, Hasson, Hendler, \& Malach, 2001; Wang, et al., 2013). The nature and extent of category effects in retinotopic cortex remains to be systematically evaluated and, here, we provide some evidence consistent with the suggestion and some existing empirical evidence that there is category tuning in early visual cortex. 


\subsection{Relationship between retinotopic and category-selective cortical regions}

That we see mixing of category and spatial position (location) effects seems incontrovertible, especially at higher levels of the visual system and probably at lower levels as well. The question, then, is how does this topography of intermixing arises. One obvious possibility is that this occurs as a result of the coupling and connectivity pattern between earlier and higher-order areas in a bidirectional network. Although, again, only few studies have investigated this, there are some hints that connectivity may play a relevant role. For example, two category-selective regions in ventral cortex - the PPA and the FFA - have been shown to exhibit an expected peripheral versus foveal bias in their connectivity with visual area hV4 (Baldassano, lordan, Beck, \& Fei-Fei, 2012). Motivated by considerations of functional network integration and the strength of the bidirectional connections in the ventral visual cortex, as well as by the growing need for a more comprehensive characterization of connectivity between early and category-specific regions, we examine category-selectivity, spatial position and their interaction from caudal to rostral regions of ventral visual cortex. Additionally, we explore the functional connectivity between these different regions.

In sum, in a cluttered scene, visual attention is thought to operate through a biased competition (Desimone \& Duncan, 1995) of visually-responsive neurons (V1, V2, etc.) that code spatially restricted (spatiotopic) information. These spatially encoded signals serve to modulate the representation in these lower-level visual areas according to the locations of a neuron's receptive field. Here, we explore spatial aspects of complex representation are also coded in highlevel visual regions of the brain, suggesting that the spatiotopic attentional modulation of these representations may directly influence high-level areas outside of the traditional retinotopic regions. Furthermore, we characterize the connectivity between the low- and high-level visual 
regions, which may play a critical role in how attention-biasing signals are propagated throughout the visual system (Greenberg, et al., 2012).

\section{Material and methods}

\subsection{Participants}

Eight right-handed subjects (4 females, mean age 51, range 25-66) with normal or corrected-tonormal vision participated in this study. Informed consent was obtained for all subjects, and the study protocol was approved by the Institutional Review Boards of George Washington University (GWU) and Carnegie Mellon University (CMU).

\section{2 fMRI acquisition}

MRI data were collected on Siemens 3-Tesla scanners at two sites, one equipped with a twelvechannel head coil at the Center for Functional and Molecular Imaging (CFMI) at Georgetown University and the other with a thirty two-channel head coil at the Scientific Brain Imaging Research center (SIBR) at Carnegie Mellon University. fMRI data were collected during a single 1.5 hour session for each participant. High-resolution anatomical images (1x1x1mm resolution) were acquired using MPRAGE T1-weighted sequence (TR=2,530ms, TE=3.5ms, field of view $=256 \times 256 \mathrm{~mm}^{2}$, matrix $=256 \times 256$, number of slices $\left.=176\right)$.

Functional images for the main experimental task were acquired with whole brain $\mathrm{T} 2{ }^{*}$ weighted echoplanar imaging (EPI) pulse sequence $(T R=2500 \mathrm{~ms}, T E=30 \mathrm{~ms}$, flip angle $=90$, field of view $=192 \times 192 \mathrm{~mm}^{2}$, matrix $=128 \times 128$, voxel size $=1.5 \times 1.5 \times 2 \mathrm{~mm}$ voxels with $0.4 \mathrm{~mm}$ gap). Thirty-six axial slices were acquired and were aligned roughly parallel to calcarine sulcus for maximal coverage of occipital, parietal, and posterior temporal cortex. In addition to the functional images 
of the main experimental task, three additional functional imaging scans were collected as separate localizers for each participant.

Location localizer images were acquired using EPI T2*-weighted gradient echo sequence (TR=2000ms, TE $=30 \mathrm{~ms}$, flip angle $=90$, field of view $=200 * 200 \mathrm{~mm}^{2}$, matrix $=64 * 64$, voxel size=3.1x3.1x4mm,number of slices=28). Retinotopic mapping was done using EPI T2*-weighted gradient echo sequence $\left(T R=3000 \mathrm{~ms}, T E=30 \mathrm{~ms}\right.$, flip angle $=90$, field of view $=200 * 200 \mathrm{~mm}^{2}$, matrix $=64 * 64$, voxel size $=3.1 \times 3.1 \times 4 \mathrm{~mm}$, number of slices=28). Lastly, the face/house localizer was collected using EPI T2*-weighted gradient echo sequence $(\mathrm{TR}=300, \mathrm{TE}=30 \mathrm{~ms}$, flip angle=90, field of view $=200 * 200 \mathrm{~mm}^{2}$, matrix $=64 * 64,3 \mathrm{~mm}$ thickness, no gap, number of slices $=35$.

\subsection{Stimuli and Procedure}

Stimuli were generated by using custom MATLAB (The Mathworks, Inc., Natick, MA) scripts with the Psychtoolbox extensions (Brainard, 1997; Pelli, 1997), and back projected onto a screen mounted at the rear end of the scanner. Subjects viewed the display via a mirror attached on the head coil, and held a single push-button in their right hand. Participants completed functional runs of the main experimental task as well as of localizer scans that permitted the independent selection of ROIs for detailed analysis.

2.3.1 Experimental task. On each trial, a single stimulus was presented at one of 4 possible locations, situated one per quadrant at equidistant eccentricity around the central fixation cross (see Figure 1). This arrangement allowed us to isolate and compare BOLD responses to stimuli in the ipsi-, contra-, upper- and lower-visual fields. The stimuli were gray scale images of faces or houses (subtending $3 \times 3^{\circ}$ of visual angle). The 4 locations $\times 2$ categories design led to eight 
conditions of interest. Examples of stimuli and their position on the screen are shown in Figures 1a-c.

To ensure that fixation was maintained, participants monitored the central fixation cross to detect color changes in the color of the fixation cross from black to green (a somewhat difficult discrimination, see below) while the task-irrelevant faces/houses were presented in one of the four possible locations in a block design. In order to increase difficulty of the fixation color change detection task, and, by extension, ensure the likelihood of participants' maintaining fixation, the time between color changes was jittered around a mean of $5 \mathrm{~s}$. Having a central fixation task also ensured that attention was equally distributed to the different locations and stimulus categories. Each run was 317.5 s long and consisted of 16 blocks that were separated with a fixation-only screen that lasted for 1.5s. Each block lasted for 16s, and contained 32 images (16 faces and 16 houses) that were presented for $300 \mathrm{~ms}$ with an inter stimulus interval of $200 \mathrm{~ms}$. Each run started with a fixation only screen for 18.25 s, and ended with final fixation for 20.75 s. Stimuli were selected from 128 grayscale house and 128 grayscale face images, with no repeated stimuli within a block. The four locations were equiprobably sampled at the beginning each block. Pilot testing outside the scanner confirmed that all subjects could perform the central fixation task with above $80 \%$ accuracy ensuring that participants maintained fixation. Each participant completed 4 experimental runs.

Because any differences in activation of the visual system, especially in early visual cortex, might arise from low-level image differences between the faces and houses, we analyzed the images along a number of dimensions, following the approach of Stigliani et al. (2015). We derived the mean Michelson contrast, which is the ratio between the difference and sum of maximum and minimum pixel intensities, and showed no difference between the faces and houses on this metric. The mean luminance of the face and house images did not differ either. To assess image similarity, 
we measured the mean Euclidean difference between the normalized grayscale values of each stimulus and every other stimulus of either same or different category. This distance was calculated for each image pair separately, within and between categories. Image similarity within the class of faces was greater than similarity within houses, but the similarity across face-house comparisons and similarity within houses were not significantly different. Last, using the Shine toolbox, we calculated the rotational average of the Fourier energy spectrum for the face and house categories separately. This analysis revealed that there was greater energy for houses than faces but only in the high spatial frequency range. For the most part, then, the statistics of the input images were largely similar. We consider, in the results section, whether any of the minimal observed differences might account for the fMRI findings.

2.3.2 Functional Localizers. In addition to the experimental task, three sets of independent localizer scans were conducted for each participant during the same scanning session (see Figure 1c): (i) Meridian localizer which localized borders between early visual cortex regions V1, V2, V3, and V4; (ii) Location localizer to identify patches of cortex responsive to the four specific stimulus locations (e.g., retinotopic area of cortex responsive to the upper left location); and (iii) Category localizer to identity face- selective and house-selective regions of cortex.

The procedures and stimuli for three localizers were as follows:

(i) During meridian mapping, subjects fixated on a central fixation point that randomly changed color to black or white and that appeared for variable duration. Subjects were required to hold down a response button when the dot was black and to release it when the dot turned white. Concurrently, a checkerboard pattern was presented in a bowtie shape that flipped independently between horizontal and vertical meridians. This method allowed mapping of borders between dorsal and ventral retinotopic regions of visual cortex (Slotnick \& Yantis, 2003) and, on this basis, 
we delineated borders between V1d, V1v, V2d, V2v, V3d, and V3v, V4d ${ }^{1}, V 4 v$. The initial fixation duration was $12 \mathrm{~s}$, each meridian was presented for $16 \mathrm{~s}$, and final fixation duration was $9 \mathrm{~s}$; total scan time was 309 seconds.

(ii) The location localizer consisted of a square of flickering checkerboards (at $4 \mathrm{~Hz}$ alternations) -- the square was the same size as the images used in the experimental task (Shomstein \& Behrmann, 2006). As in the experimental task, participants were instructed to press the response button whenever the fixation cross changed color from black to green. There were a total of 20 blocks, with each diagonal location dyad sampled 5 times (Fig 1c. middle panel). Initial fixation duration was 10 s and each location block lasted 10s, with 9s fixation between each block and a final fixation of $5 \mathrm{~s}$ with a total scan time of $386 \mathrm{~s}$.

(iii) Category-selective localizer: For the purposes of identifying regions of face- and houseselective cortex, the localizer scan included blocks of faces, houses, objects, and patterns, all of which were presented at the center of the screen. A stream of 9 stimuli were presented one at a time in a block of 9 seconds and participants performed a one-back task, identifying rare repeated images by button press. The initial fixation duration was $27 \mathrm{~s}$, the interblock fixation was $6 \mathrm{~s}$, and final fixation duration was 9s. Each participant completed a single face/house localizer scan that lasted 450seconds.

\section{4 fMRI preprocessing}

Data analysis was performed using BrainVoyager QX (version 2.3.0; Brain Innovation, Maastricht, The Netherlands) and customized MATLAB scripts. Images from each functional run were slice time corrected, motion corrected and then temporally high-pass filtered with a 128-s period

\footnotetext{
${ }^{1}$ We elected to use the term V4d and established its coordinates in the following way: after the borders were drawn on the inflated surface of cortical sheet, vertex positions were then converted to voxel locations in TAL space (left: $-25,-90.4,8.7$; right: $26.11,-89,12.8$ ). It should be noted that this region is also sometimes referred to as LO1/LO2.
} 
cutoff. All EPI and anatomical images were transformed into the standardized Talairach and Tournoux atlas space (Talairach \& Tournoux, 1988) and interpolated into $2 \mathrm{~mm}$ isotropic voxels. Localizer task data were spatially smoothed with a 3mm FWHM kernel, and data from the main task were not smoothed. The cortical surface of each participant was corrected for inhomogeneity, reconstructed by segmenting the right and left hemispheres, segmenting white from gray matter, and inflating the cortical sheet. The data were analysed using Brain Voyager and customized Matlab scripts.

\subsection{ROI selection}

We defined 11 regions of interest in each hemisphere for each participant, 8 from early visual cortex: areas V1, V2, V3 and V4 were each subdivided into a dorsal and ventral aspect, using the meridian localizer. Borders were defined between early visual areas (V1 to ventral V4) on the inflated brain and then, Patches of Interest (POIs) were drawn within the four visual areas by a separate location localizer in which flickering checkerboards $(4 \mathrm{~Hz})$ were presented at the exact locations as the four color patches in the experiment. This arrangement allowed us to isolate ipsi-, contra-, upper- and lower-visual fields for our analysis. POIs were then drawn between the vertical and horizontal meridian, thus restricting them to separate cortical areas. Last, we identified FFA and PPA, based on the category localizer, and, following recent protocols, we divided FFA into FFA1 and FFA2 (Weiner \& Grill-Spector, 2012).

Category-selective regions were identified separately in each of the left and right hemispheres for each participant using the following contrasts; FFA1 and FFA2: faces> houses (Kanwisher, McDermott, \& Chun, 1997), PPA: houses > faces (Epstein \& Kanwisher, 1998). POls were defined as clusters of at least 15 contiguous voxels exceeding an uncorrected statistical threshold of $p<0.001$. 
It is important to note that all the ROls were defined from the localizer scans and then the activation profile from the main experiment was sampled independently. Also, in the localizer scan, the higher-order regions were identified via stimulus presentation to central vision and thus the definition of category-selective region is independent of visual field.

\subsection{Univariate analysis}

For each ROI, the average Blood Oxygenation Level-Dependent (BOLD) response magnitude across the whole ROI was calculated for each condition using traditional univariate methods. In order to test the interaction between 4 stimulus positions and 2 stimulus categories, 8 conditions were used as follows: upper left face, upper left house, lower left face, lower left house, upper right face, upper right house, lower right face, and lower right house. Percent signal change of each condition for each ROI was extracted at the BOLD profile peak plus minus one time point. Data were then exported to Matlab (Mathworks) using Brain Voyager's BVQXtools Matlab toolbox, and all subsequent analyses were done in Matlab.

\subsection{Functional connectivity analysis}

Functional connectivity between early visual cortex regions and higher areas FFA1, FFA2 and PPA was analyzed as illustrated in the workflow in Figure 7. The average BOLD time course data were obtained for each POI separately in each hemisphere. Data from the 4 runs of the main experimental task were mean-centered individually and then de-trended into a single time series vector. Each time point was marked either as a fixation or experimental block that is a combination of stimulus location and category.

In order to investigate the effect of stimulus location and category on functional connectivity between early visual cortex and higher cortical areas, we first identified time points at 
which the stimulus location was in the retinotopic quadrant for each dorsal/ventral V1-V2-V3-V4. After identifying these time points with maximal response separately for each early visual cortex patch, we further divided them into two subsets for stimulus categories faces/houses. Then, we conducted correlation analyses among these subsets of time points between all pairs of early visual cortex POIs and higher cortical areas. We obtained the r-value for each pairwise comparison separately for face and house categories for each participant separately. These Pearson correlation $r$-values were Fisher transformed into z-scores for averaging across the participants and statistical testing. The task-related functional connectivity matrices in Figure 8 shows the pairwise correlations between lower and higher visual cortex regions (averaged across all participants) during stimulus categories of faces and houses, and the standard deviation of the correlations are shown in a matrix in Appendix 2.

\section{Results}

The goal of this study was to examine spatial position sensitivity and category-selective effects, as well as their interaction, both in regions of early visual cortex and in higher-order categoryselective visual cortex. In addition, we aimed to characterize the functional connectivity patterns between these regions of interest. We consider each of these in turn.

\subsection{Early visual cortex: Spatial and category effects}

A six-way analysis of variance (ANOVA) with region (V1, V2, V3 and V4), dorsal/ventral subregions, hemisphere (left, right), stimulus hemifield (left, right), stimulus position (upper, lower field) and category (face, house) was conducted with average BOLD percent signal change as the dependent measure. We discuss the differential effects of spatial location in each cortical region first, 
followed by the effects of category-selectivity. We only report the significant effects (to examine the entire $\mathrm{F}$ table, see Appendix 1).

\section{Spatial effects}

The 6-way ANOVA resulted in two significant five-way interactions but not a six-way interaction. We consider these higher-order interactions first and report the lower order effects in light of these interactions. We have used a family-wise Bonferroni correction to correct for multiple comparisons and only report those findings that survive this correction. In this section, we start by describing the significant five-way interaction that does not interact with category. The findings related to interactions with category appear below.

The five-way interaction of region $\mathrm{x}$ hemisphere $\mathrm{x}$ hemifield $\mathrm{x}$ dorsal/ventral $\mathrm{x}$ upper/lower quadrant, $(F(3,21)=18.5, p<.001)$ (see Figure 2$)$ follows the expected topographic organization of early visual cortex and replicates the well-known positional influences in early visual cortex. First, activation is greater for the contralateral than ipsilateral hemifield stimulation in the corresponding hemisphere (two way interaction: hemisphere $x$ hemifield, $(F(1,7)=199.7, p<.001)$, and stimulation in the lower and upper field result in greater ventral and dorsal cortex activation, respectively, $(F(1,7)=149.1, p<.001)$. This pattern is magnified in the right over left hemisphere (three-way interaction, hemisphere $x$ dorsal/ventral $x$ upper/lower, $F(1,7)=8.7, p<.02$ ). Unsurprisingly, these factors all interact (four-way interaction: hemisphere $\mathrm{x}$ left/right hemifield $\mathrm{x}$ dorsal/ventral $x$ upper/lower, $(F(1,7)=113.2, p<.0001)$. There are a host of lower order interactions as well but these are subsumed by the higher-order interactions described here (see Appendix for full $\mathrm{F}$ table).

When one considers the effects of stimulus position and hemisphere for each region (V1V4), there is a five-way interaction of region $\mathrm{x}$ hemisphere $\mathrm{x}$ dorsal/ventral $\mathrm{x}$ left/right hemifield $\mathrm{x}$ 
upper/lower visual field, largely reflecting the reduction of these positional influences as one moves anterior to area V4 (see Fig 2a-d, which plots the four-way interaction separately for regions V1-V4). Post-hoc comparisons ( $p<.01$ for multiple comparisons) revealed that although the same pattern was largely observed for V1-V4, a few differences were evident. For example, the greater response of dorsal and ventral cortex to stimulation in the lower and upper field, respectively, is reduced in V4 compared to V1 (n.s. to V2 and V3), (three-way interaction: dorsal/ventral $x$ upper/lower $x$ region, $F(3,12)=8.9, p<.01)$. Unsurprisingly, in light of this three-way interaction, the four-way interaction of hemisphere $\mathrm{x}$ hemifield $\mathrm{x}$ dorsal/ventral $\mathrm{x}$ upper/lower, $(F 1,7)=113.15, p<.000)$, was also significant. Taken together, the analysis of the position of the stimulus (left/right field, upper/lower field) $\mathrm{x}$ anatomical region (dorsal/ventral) $\mathrm{x}$ region reflect the presence of the positional effects in all early visual regions in both hemispheres with the diminution of positional specificity in later areas, such as V4, compared to earlier areas, such as V1. This analysis serves essentially as a sanity check, with an expected reduction in spatial selectivity given increase in receptive field sizes from areas V1 to V4, and ensures that the standard and expected organizational and positional effects can be replicated.

\section{Category effects}

In addition to the predicted effects of differential BOLD for stimuli in different spatial positions in the input, the more interesting result was the differential effect of category in these early cortical regions. An effect of category, that is a difference in BOLD signal for faces versus houses, interacted with four other factors: hemisphere $\mathrm{x}$ dorsal/ventral $\mathrm{x}$ hemifield $\mathrm{x}$ upper/lower quadrant $x$ category, $(F 1,7)=15.707, p=0.005$ (see Fig 3 plotting the greater signal for houses than faces as a function of dorsal/ventral, left/right hemifield, and upper/lower hemifield separately for left and right hemisphere). A post hoc analysis of this interaction $(p<.01$ correction) reveals that, in 
both the right and the left hemisphere, there is greater signal for houses than faces when the stimuli are presented in the lower than in the upper visual field and this is so to a greater degree in the right than left hemisphere. The only other significant comparison is the greater signal for houses than faces in the upper right visual field in the left hemisphere. This last result is unexpected and surprising and probably warrants replication and further examination. Together, this five-way interaction result reveals the effect of category even within early visual cortex, where, collapsed across region, houses elicited greater response than faces especially in the lower visual field.

There is also a significant four-way interaction of category $\mathrm{x}$ region $\mathrm{x}$ dorsal/ventral $\mathrm{x}$ hemifield, $(F 3,21)=4.073, p=0.02$, which results from the stronger BOLD response to houses than to faces, especially as one proceeds from V1 through to V4, and this is so to a greater degree for stimuli in the lower than upper field and in the right over left hemisphere. There was also a significant interaction of dorsal/ventral portion of visual cortex $x$ hemifield $x$ category, $[F(1,7)=$ 15.596, $p=.006$ ] with disproportionately greater activation for houses than for faces in the left hemifield in the dorsal regions than in ventral regions in both hemispheres. A three-way interaction of dorsal/ventral $\mathrm{x}$ stimulus position (top/bottom) $\mathrm{x}$ category supports the same finding with greater house than face activation in dorsal visual cortices when the stimuli appear in the lower visual field, $[F(1,7)=7.25, p<.05]$.

Together, these findings indicate the presence of both position and category effects in regions V1 through V4. Not only do we observe the expected spatially specific effects associated with the anatomical constraints of the visual system (for example, contralateral > ipsilateral advantage in each hemisphere and dorsal and ventral mapped to lower and upper stimuli, respectively) but we also see some effects of category with, on the whole, larger signal for houses than faces especially when presented in the lower visual field, as reflected in dorsal regions of V1- 
V4, and with greater effect as one proceeds from V1 through to V4. Interestingly, it appears that as positional specificity decreases, category specificity increases as information proceeds to more anterior extrastriate regions.

As will be elaborated on below, the manifestation of category effects in early visual cortex may be a consequence of the functional connectivity of the system. Before reaching this conclusion, however, it is important to rule out two alternative explanations, one concerning the differences we have noted in the statistics of the face/house images and the other concerning differential eye movements for the two categories. As indicated previously, faces are more similar to each other than houses and houses contain greater high frequency power than do faces. Whether either of these differences is at play is not clear - perhaps the most relevant finding is that the category effect on early visual cortical function is not apparent under all conditions: for example, we only see the greater activation for houses than faces in the lower visual field but not in the upper field and the category effect is greater as one moves more rostral in the system. These particular and specific patterns of category differences cannot easily be accommodated by main effects of differences in image statistics of faces versus houses and, thus, we think it unlikely that the patterns in low-level cortex are explained by image properties.

A second potential explanation concerns differential patterns of eye movements. Even though the central target detection task was specifically designed to prevent eye movements, it is possible that there were eye movements toward the face/house stimuli, perhaps even differentially by category. We reasoned that if participants made eye movements toward the images from the categories, it would impact accuracy on the central fixation task. Thus, if eyemovements drive the observed category differences, then potentially, those who maintained fixation well should show a different profile from those who maintained fixation less well. To this end, we examined the accuracy of the participants on the fixation task, which was designed to 
ensure that fixation was maintained. Accuracy was greater than $80 \%$ for all participants and close to $90 \%$ for the majority. To explore whether the ability to maintain fixation influenced the fMRI pattern, we re-ran the six-way ANOVA with an additional factor of group, defined by a median split in fixation task. There was neither a main effect of group ( $p>.05)$ nor any significant interactions of group and any other variable/s (all $p>.05$ ). While the power in the analysis described above is not large owing the small groups of participants ( $n=4$ for each split half), again, the specific pattern of findings would somehow need to be accommodated by an explanation of differential eye movements and it is not apparent how such an explanation can account for the greater activation for houses over faces to a greater degree in the lower than upper visual field. We think, therefore, that it is unlikely that eye movement differences can explain the particular set of findings from early visual cortex.

\subsection{Higher-order cortex: Spatial and category effects}

Initially, two category-selective regions, one corresponding to the FFA and one to the PPA, were defined per individual in each hemisphere. In light of the growing recognition that FFA may be subdivided, we further separated the face-selective region in the fusiform into FFA1 (more posterior) and FFA2 (more anterior) using existing guidelines and criteria for division (Weiner \& Grill-Spector, 2012). The ANOVAs are therefore performed using percent signal change as the dependent measure and region (FFA1, FFA2 and PPA), hemisphere (left, right), hemifield (left, right), stimulus position (upper, lower quadrant) and category (face, house) as the within-subject factors. As above, our interest is in sensitivity to stimulus position and category selectivity in these three regions of cortex. Because we expect to observe category selectivity in these regions, we first reports effects of category and ensure that we can replicate previous findings, and then we go on to discuss effects of and any interactions with positional specificity. 
Category effects

Collapsed across hemispheres, there was a stronger BOLD response to faces than houses, [category: $(F 1,7)=9.4, p=.018]$. Additionally, FFA1 and FFA2 responded more strongly to faces than houses and PPA responded more strongly to houses than faces, [two-way: region x category, $F(2,14)=63.03, p<.000]$ (as reflected in Fig 4). There is, however, differential selectivity for faces versus houses as a function of region and hemisphere, as revealed in a significant three-way interaction of region $x$ hemisphere $x$ category, $[F(2,14)=18.3, p<.000]$; while both hemispheres showed greater activation for faces than houses in FFA1 and FFA2 along with the reverse pattern in PPA, the difference between face and house activation trended to be stronger in the RH than $\mathrm{LH}$ in FFA2 than in FFA1 $(p=.05)$. This result suggests that the well-known right hemisphere lateralization of FFA may be somewhat more strongly driven by the activation profile of FFA2 than of FFA1. There was also a trend for house activation to be stronger in FFA1 and in FFA2 than was face activation in PPA. Consistent with this, there was disproportionately greater activation overall for faces than for houses in the right than left hemisphere, (hemisphere $x$ category, $[F(1,7)=8.05$, $p=.025]$. There were no other significant effects. These results are consistent with decades of research reporting face selectivity in FFA, and house selectivity in PPA with magnification of this difference in the $\mathrm{RH}$ over $\mathrm{LH}$.

\section{Spatial effects}

The first result we explore is the presence of an interaction of hemisphere $x$ hemifield, $(F(1$, 7)=26.4, $p<.001)$, reflecting the increased activation in each hemisphere from contralateral versus ipsilateral stimuli (see Fig. 5). The interaction of cortical region with the spatial position of the stimulus was evident in the interaction between region $\mathrm{x}$ hemisphere $\mathrm{x}$ hemifield (left,right) $\mathrm{x}$ 
category, $[F(2,14)=21.4, p<.000]$. An examination of the findings reveals the strongest response to faces compared with houses in the LVF in both RH FFA1 and FFA2 but stronger responses to faces than houses in the RVF in FFA2 than in FFA1 in the RH, reflecting less spatial specificity in FFA2 than FFA1. In the PPA, there was a stronger response in the $\mathrm{RH}$ to houses than faces shown in the LVF but equally strong BOLD to houses than faces in the LH for stimuli in the right and left visual fields.

Last, although not surviving Bonferroni correction, there is a trend for a significant fourway interaction of region $x$ hemisphere $x$ hemifield $x$ upper/lower quadrant interaction, $(F(2$, $14)=3.8, p=.05$ ) (see Fig 6 ). A post hoc comparison ( $p<.01$ for correction) reveals no differential sensitivity to upper versus lower quadrants in either visual field in either hemisphere in FFA1 and FF2 but in PPA, there was greater sensitivity to upper quadrant stimuli in the contralateral visual field i.e. greater in RVF manifest in LH and greater in LVF manifest in RH. These effects hold for both face and house trials in the PPA, hence the absence of the higher-order interaction with category.

Taken together, these findings confirm the expected category effects in higher-order visual areas with greater selectivity for faces in FFA1 and FFA2 and for houses in PPA, especially over the $\mathrm{RH}$. Of perhaps greater interest is the effect of spatial position of the stimulus on the cortical BOLD profile in these higher-order areas. There is increased activation in each hemisphere from contralateral versus ipsilateral stimuli. Of interest, there is less spatial specificity in FFA2 than in FFA1 and the LH PPA is less spatially tuned in the response to houses than is the RH PPA. Last, the PPA has greater sensitivity to upper quadrant stimuli in the contralateral than ipsilateral visual field whereas the FFA is not selectively activated by stimuli in the upper versus lower fields.

\subsection{Functional connectivity between ventral and early visual cortex}


Thus far, we have shown coupling of spatial and category effects both in early and later regions of visual cortex. As suggested in the Introduction, in a system with bidirectional connectivity, this might not be that surprising as graded effects of both variables may be present through the visual system with spatial effects holding greater sway in earlier regions category effects holding greater sway in later regions. One obvious way in which these seemingly disparate effects might be instantiated is by virtue of functional connectivity between the earlier and later regions of the visual system. To explore this, we evaluated the functional connectivity between early and higher visual areas during the experimental task. This involved correlating the signal from regions V1-V4 with the signal from FFA1, FFA2 and PPA (see Fig 7 for schematic of the adopted approach). For each subject, we calculated the functional correlation between early and higher visual areas for face and house categories separately. The correlation values from each subject is included in Appendix 2. We then transformed these $r$ values into z-scores and averaged over subjects. Given the numerous correlations performed, we only report those correlations that exceed $p<.001$ ( $z>0.440$ ) (but the full z-scored correlation matrix is shown in Fig 8 and the raw correlations from subjects are included in Appendix 2).

When faces were presented as stimuli, the BOLD signal in left FFA1 was significantly correlated with the ventral but not dorsal aspects of the left hemisphere early visual cortical regions (V1 through V4). The same pattern held in the RH: the right FFA1 was significantly correlated with the ventral but not dorsal aspects of the right hemisphere early visual cortex (V1 through V4). The functional connectivity from the contralateral V1-V4 ventral aspects approached significance in both hemispheres, as well, but were not as strongly connected as the ipsilateral regions. There was also a trend for the right PPA to be connected to the right early visual regions during face trials and the signal from the left PPA was significantly correlated with just the left hemisphere V4 ventral region. 
When houses were presented, as was the case with faces, the BOLD signal in left FFA1 was correlated with left hemisphere early visual cortex (V1 through V4) ventral but not dorsal regions. There were no correlations with the right FFA1 or either left or right FFA2. The only other significant correlations were between the right PPA with the right hemisphere V2-V4 ventral regions.

Several findings are of particular interest. The first is that for both face and house trials, the earlier and later areas that are correlated are ipsilateral, rather than contralateral. This may seem surprising given the weak known crossing of signals contralaterally but the functional connectivity measures are more likely to be reflecting intra-hemispheric constraints imposed by direct structural connections. It is also worth noting that all functional connections observed are between ventral early visual regions and no correlations are noted with dorsal regions. The second key observation is that FFA1 but not FFA2 is correlated with earlier areas and this is consistent with the analyses from higher-order regions (see section 3.2) revealing less spatial specificity in FFA2 than in FFA1. Last, left, but not right, PPA is correlated with left V4 ventral both in response to faces and in response to houses, perhaps reflecting the reduced category selectivity in the LH compared with the $\mathrm{RH}$, again, as observed in the univariate analyses.

A brief examination of the correlation matrix, shown in Figure 8, might suggest that regions that are more anatomically proximal may be more highly correlated. Close scrutiny of the data reveal that this is not the case. For example, the correlation between left FFA1 and lh_v2v is almost same as Ih_v4v (0.61 versus 0.62$)$. Similarly, right FFA1 and rh_v1v correlation is the same as rh_v4v and the right PPA functional connectivity is the same with rh_v3v and rh_v4v. It appears, therefore, that anatomical proximity is not driving the strength of the functional correlations here. 


\section{Discussion}

The goal of this paper was to examine, using functional MR imaging of early and late visual cortices, the ramifications of a bidirectionally connected visual system. The standard conceptualization of the visual system has been one in which earlier parts of visual cortex encode properties of the incoming stimuli, such as their spatial location, viewpoint and pose, and that this encoding is largely enabled by the small receptive field sizes of neurons in regions V1, in particular but also in V2-V4. Then, as one proceeds more rostrally, the claim has been that areas of the visual system represented information abstracted away from the spatial properties of the incoming stimuli and increasingly tuned to more abstract representations of, for example, category types like faces versus houses. More recently, with deeper understanding of the functional and anatomical properties of the ventral visual system (see (Grill-Spector \& Weiner, 2014; Kravitz, et al., 2013; Weiner \& Grill-Spector, 2012; Weiner \& Zilles, 2015)), the current consensus is that the ventral visual pathway is a recurrent and highly interactive occipitotemporal network linking early visual areas and later regions, perhaps as far rostral as the anterior temporal lobe.

To characterize the graded effects of both spatial position and category membership of a visual stimulus on cortical activation profiles, participants viewed two displays from two categories of stimuli (faces and houses) with individual stimuli appearing in the upper or lower quadrant of the right or left visual field. Although participants did not respond to the stimuli directly, and were simply performing a fixation change detection task, significant effects of position and of category type were observed in both early and later parts of the visual system. Specifically, in addition to the expected mapping of stimuli in upper and lower visual fields to ventral and dorsal aspects of regions V1-V4, and the expected mapping of greater activation to contralateral than to ipsilateral stimuli in regions V1-V4, we also documented greater signal for houses than faces when the stimuli are presented in the lower than in the upper visual field and this was so to a greater degree 
in the right than left hemisphere and in dorsal rather than ventral regions. Note that this difference cannot simply be attributed to low-level differences between faces and house; if this were the case, one would expect to observe similar effects in upper and lower visual field. Thus, the difference that we observed is likely indicative of the category specificity that is differentially represented in the upper and lower visual field. By the same token, in higher-order areas, FFA1, FFA2 and PPA, we not only replicated the standard category-selective effects with activation for faces greater than for houses in FFA1 and FFA2 (and the converse in PPA), but we also observed some spatial specificity, as well. Notably, FFA2 responded in a less spatially-specific fashion than FFA1. Also, although there was no differential sensitivity to upper versus lower quadrants in either visual field in either hemisphere in FFA1 and FFA2 but, there was greater sensitivity to upper quadrant stimuli in the contralateral visual field in PPA.

The finding that higher-order areas are constrained by spatial position is consistent with the large number of recent studies that have shown that object representations in higher-order regions retain position specificity. For example, Kravitz et al. (Kravitz, et al., 2010) provided converging evidence with both behavior and fMRI investigations that visual object representations are position-dependent. In their behavioral study, visual object priming was significant only when the prime and probe shared spatial position and any shifts in position between prime and probe resulted in less priming. Neuroimaging uncovered the neural correlates of the behavioral finding, revealing that object representations in object-selective cortex also demonstrated the positiondependence of object representations (Arcaro, et al., 2009; Kravitz, et al., 2010; Schwarzlose, et al., 2008; Strother, et al., 2010).

The finding that lower-order areas are affected by stimulus type (category) and that this cannot simply be a product of differences in image statistics nor a product of differential eye movement patterns for the two categories. We observed greater signal for houses than faces 
when the stimuli were presented in the lower than in the upper visual field and this was so to a greater degree in the right than left hemisphere. This particular pattern of findings cannot be easily accommodated by an account based on image statistics or eye movements. Nevertheless, we characterized the images along a host of dimensions (luminance, contrast, spatial frequency) and observed only minimal differences between the face and house images and when we did see the difference, the direction varied: greater similarity within faces than within houses but greater power in high spatial frequencies for houses than faces. We also showed that there was no difference in functional activation profile for those who maintained fixation well (i.e. made few eye movements) and those who did not, making it unlikely that the category differences arise from an eye movement artifact.

Last, we mapped clear functional connectivity profiles between higher order areas FFA1 and PPA but not FFA2, with early visual areas. Of note the connectivity was stronger for ipsilateral, within hemisphere coupling rather than with contralateral connectivity. These results implicate functional connectivity as the possible basis for the intermixing of position and category effects in a distributed fashion along the entire ventral pathway and likely reflect the direct structural connectivity intrahemispherically rather than interhemispherically.

Together, these data suggest surprisingly systematic topological arrangement of functional representations in visual cortex. In particular, we suggest that the presence of both spatial position and category effects in both early and later cortex as well as connectivity between these regions may be a direct product of the bidirectional connectivity of the ventral visual system and the abundant feedforward and feedback connections (Felleman \& Van Essen, 1991; Van Essen, 1992). Such a system may well be advantageous as a network that bridges early visual cortex and higher order regions can support more complex computations and representations. 
It does remain a possibility that the presence of both spatial and category effects in lower order cortical regions may be a product of some sub-population of neurons in V1-V4 being sensitive to house/face features. The functional connectivity measures, however, suggest that the outcome may not simply be a function of computations performed in just early or just late visual cortical regions but rather the intermixing of these effects might be a product of the reciprocal connectivity between the regions. Further research is needed to clarify the source of the intermixing of category and spatial information more precisely.

A final question one might ask is where this topological organization and connectivity comes from. Numerous recent papers have addressed this issue and have considered factors such as clustering of neurons with similar properties, organization relative to cortical folding patterns, and superimposition of multiple functional representations on the same cortical expanse as well as anatomical constraints on functional topologies (Grill-Spector \& Weiner, 2014; Weiner \& GrillSpector, 2012). It is also possible, however, that the representation of an object might be affected by the statistics of its appearance on the retina and be a functional outcome of real-world experience with the visual world. For example, Wang et al. (Wang, et al., 2013) report differential sensitivity of FFA and PPA to stimuli in the meridian and eccentric dimensions and argue that these different processing strategies might depend on the retinal position at which faces or houses are typically observed in daily life. As such, long-term experience might affect large scale cortical organization producing topographic effects in ventral visual cortex (Levy, Hasson, Avidan, Hendler, \& Malach, 2001; Malach, Levy, \& Hasson, 2002). Moreover, although long-term experience may play a role, these constraints and patterns of functional connectivity may be in place even without experience. Thus, because the connectivity basis for visual cortex large-scale topographical organization is present in individuals who are congenitally blind, this organization of the early-later visual cortex can develop without any visual experience (Striem-Amit, et al., 2015). 
A number of open issues remain. One immediate question is whether the findings we have observed would apply to other categories as well. Faces and houses are well known to be markedly in contrast and the selectivity in FFA and PPA is clearly documented. Whether other categories that are not as clearly opposed would yield category effects in early regions remains to be determined. Additionally, although the findings are suggestive of constraints by structural connectivity (ipsilateral greater than contralateral), this remains to be demonstrated.

In conclusion, the evidence we have presented is consistent with a dynamic interactive account of cortical organization in which regions are not isolated by firewalls. Rather, by virtue of reciprocal connectivity (functional and/or structural), effects that are considered signatures of lower-level visual cortex and effects that are considered signatures of higher-level visual cortex appear to be graded but ubiquitous, as might be expected from a bidirectionally connected system.

Acknowledgements: This work was supported by a grant from the National Institutes of Health to SS and MB (EY021644), a grant from the National Science Foundation to MB (BCS0923763), and a grant from the National Science Foundation to SS (BCS-1534823). Scanning at Georgetown's CFMI is supported by IDDRC grant 1P30HD4067. AG was supported on a training grant from the National Institutes of Health (PI: L. Reder). We thank Julie Golomb, John VanMeter, and Jeongmi Lee for their assistance in preparing this manuscript.

Note: Fatma Uyar has moved to the University of Pittsburgh and Adam Greenberg is now at the University of Wisconsin-Milwaukee. 


\section{Figure Legends:}

Figure 1: a. Trial sequence of houses and words with fixation change. b. Illustration of four locations sampled during a block of trials. c. Displays from three localizers mapping the horizontal and certical meridians, mapping of the four locations in which stimuli appear in experimental blocks and example images of faces, houses, objects and patterns for localizer of higher-level regions (with focus on face/house localization).

Figure 2: Percent signal change for stimuli presented in the left and right hemifield in the upper and lower quadrants in the left and right hemispheres as a function of dorsal and ventral cortex, plotted in regions V1-V4 separately.

Figure 3: Percent signal change that is greater for houses than faces as a function of quadrant and hemifield plotted separately for the left and right hemispheres.

Figure 4: Percent signal change for faces and houses in the left and right hemispheres plotted separately for FFA1, FFA2 and PPA.

Figure 5: Percent signal change that is greater for faces than houses in the left and right hemispheres as a function of visual hemifield, plotted separately for regions FFA1, FFA2 and PPA.

Figure 6: Percent signal change greater for stimuli in lower than upper visual field in the left and right visual fields as a function of hemisphere, plotted separately for regions FFA1, FFA2 and PPA.

Figure 7: The workflow depicting the analysis of functional connectivity.

Figure 8: Correlation matrix, derived separately for house trials and face trials, reflecting the functional connectivity values between early and higher-order visual cortical regions. 


\section{$\underline{\text { References }}$}

Arcaro, M. J., McMains, S. A., Singer, B. D., \& Kastner, S. (2009). Retinotopic organization of human ventral visual cortex. J Neurosci, 29, 10638-10652.

Baldassano, C., Iordan, M. C., Beck, D. M., \& Fei-Fei, L. (2012). Voxel-level functional connectivity using spatial regularization. Neuroimage, 63, 1099-1106.

Brainard, D. H. (1997). The Psychophysics Toolbox. Spatial Vision, 10, 433-436.

Desimone, R., \& Duncan, J. (1995). Neural mechanisms of selective visual attention. Annual Review of Neuroscience, 18, 193-197.

Desimone, R., \& Gross, C. G. (1979). Visual areas in the temporal cortex of the macaque. Brain Res, 178, 363-380.

DiCarlo, J. J., \& Maunsell, J. H. (2003). Anterior inferotemporal neurons of monkeys engaged in object recognition can be highly sensitive to object retinal position. $J$ Neurophysiol, 89, 3264-3278.

Epstein, R., \& Kanwisher, N. (1998). A cortical representation of the local visual environment. Nature, 392, 598-601.

Felleman, D. J., \& Van Essen, D. C. (1991). Distributed hierarchical processing in the primate cerebral cortex. Cerebral Cortex, 1, 1-47.

Furl, N. (2015). Structural and effective connectivity reveals potential network-based influences on category-sensitive visual areas. Front Hum Neurosci, 9, 253.

Golomb, J. D., \& Kanwisher, N. (2012). Higher level visual cortex represents retinotopic, not spatiotopic, object location. Cereb Cortex, 22, 2794-2810.

Greenberg, A. S., Verstynen, T., Chiu, Y. C., Yantis, S., Schneider, W., \& Behrmann, M. (2012). Visuotopic cortical connectivity underlying attention revealed with white-matter tractography. J Neurosci, 32, 2773-2782.

Grill-Spector, K., \& Weiner, K. S. (2014). The functional architecture of the ventral temporal cortex and its role in categorization. Nat Rev Neurosci, 15, 536-548.

Hemond, C. C., Kanwisher, N. G., \& Op de Beeck, H. P. (2007). A preference for contralateral stimuli in human object- and face-selective cortex. PLoS One, 2, e574.

Hong, H., Yamins, D. L., Majaj, N. J., \& DiCarlo, J. J. (2016). Explicit information for category-orthogonal object properties increases along the ventral stream. Nat Neurosci.

Hung, C. P., Kreiman, G., Poggio, T., \& DiCarlo, J. J. (2005). Fast readout of object identity from macaque inferior temporal cortex. Science, 310, 863-866.

Kanwisher, N., McDermott, J., \& Chun, M. M. (1997). The fusiform face area: a module in human extrastriate cortex specialized for face perception. J Neurosci, 17, 4302-4311.

Kobatake, E., \& Tanaka, K. (1994). Neuronal selectivities to complex object features in the ventral visual pathway of the macaque cerebral cortex. Journal of Neurophysiology, 71, 856-867.

Kravitz, D. J., Kriegeskorte, N., \& Baker, C. I. (2010). High-level visual object representations are constrained by position. Cereb Cortex, 20, 2916-2925. 
Kravitz, D. J., Saleem, K. S., Baker, C. I., Ungerleider, L. G., \& Mishkin, M. (2013). The ventral visual pathway: an expanded neural framework for the processing of object quality. TRENDS in Cognitive Sciences, 17, 26-49.

Levy, I., Hasson, U., Avidan, G., Hendler, T., \& Malach, R. (2001). Center-periphery organization of human object areas. Nat Neurosci, 4, 533-539.

Levy, I., Hasson, U., Hendler, T., \& Malach, R. (2001). Center-periphery organization of human object areas. Nat Neurosci, 4, 533-539.

Malach, R., Levy, I., \& Hasson, U. (2002). The topography of high-order human object areas. Trends Cogn Sci, 6, 176-184.

Matsuzaki, N., Schwarzlose, R. F., Nishida, M., Ofen, N., \& Asano, E. (2015). Upright facepreferential high-gamma responses in lower-order visual areas: evidence from intracranial recordings in children. Neuroimage, 109, 249-259.

McKyton, A., \& Zohary, E. (2007). Beyond retinotopic mapping: the spatial representation of objects in the human lateral occipital complex. Cerebral Cortex, 17, 1164-1172.

Niemeier, M., Goltz, H. C., Kuchinad, A., Tweed, D. B., \& Vilis, T. (2005). A contralateral preference in the lateral occipital area: sensory and attentional mechanisms. Cereb Cortex, 15, 325-331.

Pelli, D. G. (1997). The VideoToolbox software for visual psychophysics: Transforming numbers into movies. Spatial Vision, 10, 437-442.

Rajimehr, R., Bilenko, N. Y., Vanduffel, W., \& Tootell, R. B. (2014). Retinotopy versus face selectivity in macaque visual cortex. J Cogn Neurosci, 26, 2691-2700.

Roth, Z. N., \& Zohary, E. (2015). Position and Identity Information Available in fMRI Patterns of Activity in Human Visual Cortex. J Neurosci, 35, 11559-11571.

Sayres, R., \& Grill-Spector, K. (2008). Relating retinotopic and object-selective responses in human lateral occipital cortex. J Neurophysiol, 100, 249-267.

Schwarzlose, R. F., Swisher, J. D., Dang, S., \& Kanwisher, N. (2008). The distribution of category and location information across object-selective regions in human visual cortex. Proc Natl Acad Sci U S A, 105, 4447-4452.

Shigihara, Y., \& Zeki, S. (2014). Parallel processing of face and house stimuli by V1 and specialized visual areas: a magnetoencephalographic (MEG) study. Front Hum Neurosci, 8, 901.

Shomstein, S., \& Behrmann, M. (2006). Cortical systems mediating visual attention to both objects and spatial locations. Proceedings of the National Academy of Sciences of the United States of America, 103, 11387-11392.

Slotnick, S. D., \& Yantis, S. (2003). Efficient acquisition of human retinotopic maps. Human Brain Mapping, 18, 22-29.

Stigliani, A., Weiner, K. S., \& Grill-Spector, K. (2015). Temporal processing capacity in high-level visual cortex is domain-specific. in revision.

Striem-Amit, E., Ovadia-Caro, S., Caramazza, A., Margulies, D. S., Villringer, A., \& Amedi, A. (2015). Functional connectivity of visual cortex in the blind follows retinotopic organization principles. Brain, 138, 1679-1695.

Strother, L., Aldcroft, A., Lavell, C., \& Vilis, T. (2010). Equal degrees of object selectivity for upper and lower visual field stimuli. J Neurophysiol, 104, 2075-2081.

Talairach, J., \& Tournoux, P. (1988). Co-planar stereotaxic atlas of the human brain. New York: George Thieme Verlag. 
Van Essen, D. C., Anderson, C.H., Felleman, D.J. (1992). Information processing in the primate visual system: An integrated systems perspective. Science, 225, 419423.

Wang, W. F., Yan, T., Chen, K., Imajyo, S., Ohno, S., \& Kanazawa, S. (2013). Regional Neural Response Differences in the Determination of Faces or Houses Positioned in a Wide Visual Field. PLoS One, 8, 1-13.

Weiner, K. S., \& Grill-Spector, K. (2012). The improbable simplicity of the fusiform face area. Trends Cogn Sci, 16, 251-254.

Weiner, K. S., \& Zilles, K. (2015). The anatomical and functional specialization of the fusiform gyrus. Neuropsychologia. 


\section{Appendix 1}

\section{F-Table from six-way Analysis of Variance using percent signal change in retinotopic regions}

\begin{tabular}{|c|c|c|c|c|c|}
\hline Source & $\begin{array}{l}\text { Type III Sum of } \\
\text { Squares }\end{array}$ & df & Mean Square & $\mathrm{F}$ & Sig. \\
\hline region & 0.382 & 3 & 0.127 & 3.058 & 0.051 \\
\hline Error(region) & 0.874 & 21 & 0.042 & & \\
\hline hemisphere & 0.16 & 1 & 0.16 & 1.819 & 0.219 \\
\hline Error(hemisphere) & 0.615 & 7 & 0.088 & & \\
\hline dorsal_ventral & 0.156 & 1 & 0.156 & 1.038 & 0.342 \\
\hline Error(dorsal_ventral) & 1.055 & 7 & 0.151 & & \\
\hline left_rightHemi & 0.006 & 1 & 0.006 & 0.059 & 0.815 \\
\hline Error(left_rightHemi) & 0.724 & 7 & 0.103 & & \\
\hline upper_lowerVF & 2.407 & 1 & 2.407 & 6.54 & 0.038 \\
\hline Error(upper_lowerVF) & 2.577 & 7 & 0.368 & & \\
\hline category & 0.07 & 1 & 0.07 & 0.34 & 0.578 \\
\hline Error(category) & 1.438 & 7 & 0.205 & & \\
\hline region * hemisphere & 0.078 & 3 & 0.026 & 0.576 & 0.637 \\
\hline Error(region*hemisphere) & 0.95 & 21 & 0.045 & & \\
\hline region * dorsal_ventral & 0.103 & 3 & 0.034 & 0.607 & 0.618 \\
\hline Error(region*dorsal_ventral) & 1.186 & 21 & 0.056 & & \\
\hline hemisphere $*$ dorsal_ventral & 0.218 & 1 & 0.218 & 3.372 & 0.109 \\
\hline Error(hemisphere*dorsal_ventral) & 0.452 & 7 & 0.065 & & \\
\hline region * hemisphere * dorsal_ventral & 0.295 & 3 & 0.098 & 1.665 & 0.205 \\
\hline Error(region*hemisphere*dorsal_ventral) & 1.242 & 21 & 0.059 & & \\
\hline region * left_rightHemi & 0.213 & 3 & 0.071 & 0.954 & 0.433 \\
\hline Error(region*left_rightHemi) & 1.561 & 21 & 0.074 & & \\
\hline hemisphere * left_rightHemi & 92.006 & 1 & 92.006 & 199.725 & 0 \\
\hline Error(hemisphere*left_rightHemi) & 3.225 & 7 & 0.461 & & \\
\hline region $*$ hemisphere $*$ left_rightHemi & 0.554 & 3 & 0.185 & 3.283 & 0.041 \\
\hline Error(region*hemisphere*left_rightHemi) & 1.18 & 21 & 0.056 & & \\
\hline dorsal_ventral * left_rightHemi & 0.445 & 1 & 0.445 & 2.458 & 0.161 \\
\hline Error(dorsal_ventral*left_rightHemi) & 1.266 & 7 & 0.181 & & \\
\hline region * dorsal_ventral * left_rightHemi & 0.109 & 3 & 0.036 & 0.663 & 0.584 \\
\hline Error(region*dorsal_ventral*left_rightHemi) & 1.148 & 21 & 0.055 & & \\
\hline hemisphere * dorsal_ventral * left_rightHemi & 0.033 & 1 & 0.033 & 0.079 & 0.787 \\
\hline Error(hemisphere*dorsal_ventral*left_rightHemi) & 2.93 & 7 & 0.419 & & \\
\hline region $*$ hemisphere $*$ dorsal_ventral * left_rightHemi & 0.377 & 3 & 0.126 & 1.645 & 0.209 \\
\hline Error(region*hemisphere*dorsal_ventral*left_rightHemi) & 1.605 & 21 & 0.076 & & \\
\hline region * upper_lowerVF & 0.288 & 3 & 0.096 & 1.267 & 0.311 \\
\hline Error(region*upper_lowerVF) & 1.594 & 21 & 0.076 & & \\
\hline
\end{tabular}




\begin{tabular}{|c|c|c|c|c|c|}
\hline hemisphere * upper_lowerVF & 0.926 & 1 & 0.926 & 8.049 & 0.025 \\
\hline Error(hemisphere*upper_lowerVF) & 0.806 & 7 & 0.115 & & \\
\hline region $*$ hemisphere $*$ upper_lowerVF & 0.248 & 3 & 0.083 & 1.15 & 0.352 \\
\hline Error(region*hemisphere*upper_lowerVF) & 1.508 & 21 & 0.072 & & \\
\hline dorsal_ventral * upper_lowerVF & 106.939 & 1 & 106.939 & 149.091 & 0 \\
\hline Error(dorsal_ventral*upper_lowerVF) & 5.021 & 7 & 0.717 & & \\
\hline region * dorsal_ventral * upper_lowerVF & 1.891 & 3 & 0.63 & 8.867 & 0.001 \\
\hline Error(region*dorsal_ventral*upper_lowerVF) & 1.493 & 21 & 0.071 & & \\
\hline hemisphere * dorsal_ventral * upper_lowerVF & 0.654 & 1 & 0.654 & 8.706 & 0.021 \\
\hline Error(hemisphere*dorsal_ventral*upper_lowerVF) & 0.526 & 7 & 0.075 & & \\
\hline region $*$ hemisphere $*$ dorsal_ventral $*$ upper_lowerVF & 0.44 & 3 & 0.147 & 1.653 & 0.207 \\
\hline Error(region*hemisphere*dorsal_ventral*upper_lowerVF) & 1.864 & 21 & 0.089 & & \\
\hline left_rightHemi * upper_lowerVF & 0.062 & 1 & 0.062 & 0.263 & 0.624 \\
\hline Error(left_rightHemi*upper_lowerVF) & 1.648 & 7 & 0.235 & & \\
\hline region * left_rightHemi * upper_lowerVF & 0.261 & 3 & 0.087 & 1.109 & 0.368 \\
\hline Error(region*left_rightHemi*upper_lowerVF) & 1.651 & 21 & 0.079 & & \\
\hline hemisphere * left_rightHemi * upper_lowerVF & 0.973 & 1 & 0.973 & 2.657 & 0.147 \\
\hline Error(hemisphere*left_rightHemi*upper_lowerVF) & 2.564 & 7 & 0.366 & & \\
\hline region $*$ hemisphere $*$ left_rightHemi $*$ upper_lowerVF & 0.646 & 3 & 0.215 & 2.935 & 0.057 \\
\hline Error(region*hemisphere*left_rightHemi*upper_lowerVF) & 1.541 & 21 & 0.073 & & \\
\hline dorsal_ventral * left_rightHemi * upper_lowerVF & 0.367 & 1 & 0.367 & 5.825 & 0.047 \\
\hline Error(dorsal_ventral*left_rightHemi*upper_lowerVF) & 0.441 & 7 & 0.063 & & \\
\hline $\begin{array}{l}\text { region * dorsal_ventral } * \text { left_rightHemi } * \text { upper_lowerVF } \\
\text { Error(region*dorsal_ventral*left_rightHemi*upper_lowerVF }\end{array}$ & 0.419 & 3 & 0.14 & 1.618 & 0.215 \\
\hline hemisphere $*$ dorsal_ventral $*$ left_rightHemi $*$ & 1.812 & 21 & 0.086 & & \\
\hline $\begin{array}{l}\text { upper_lowerVF } \\
\text { Error(hemisphere*dorsal ventral*left rightHemi*upper lo }\end{array}$ & 99.769 & 1 & 99.769 & 113.155 & 0 \\
\hline $\begin{array}{l}\text { werVF) } \\
\text { region } * \text { hemisphere } * \text { dorsal_ventral } * \text { left_rightHemi } *\end{array}$ & 6.172 & 7 & 0.882 & & \\
\hline $\begin{array}{l}\text { upper_lowerVF } \\
\text { Error(region*hemisphere*dorsal_ventral*left_rightHemi*up }\end{array}$ & 3.926 & 3 & 1.309 & 18.507 & 0 \\
\hline per_lowerVF) & 1.485 & 21 & 0.071 & & \\
\hline region * category & 0.062 & 3 & 0.021 & 0.429 & 0.735 \\
\hline Error(region*category) & 1.007 & 21 & 0.048 & & \\
\hline hemisphere * category & 0.312 & 1 & 0.312 & 5.164 & 0.057 \\
\hline Error(hemisphere*category) & 0.423 & 7 & 0.06 & & \\
\hline region $*$ hemisphere * category & 0.094 & 3 & 0.031 & 1.466 & 0.252 \\
\hline Error(region*hemisphere*category) & 0.447 & 21 & 0.021 & & \\
\hline dorsal_ventral * category & 0.002 & 1 & 0.002 & 0.003 & 0.955 \\
\hline Error(dorsal_ventral*category) & 3.435 & 7 & 0.491 & & \\
\hline region * dorsal_ventral * category & 0.036 & 3 & 0.012 & 0.48 & 0.699 \\
\hline Error(region*dorsal_ventral*category) & 0.523 & 21 & 0.025 & & \\
\hline hemisphere * dorsal_ventral * category & 0.391 & 1 & 0.391 & 5.535 & 0.051 \\
\hline Error(hemisphere*dorsal_ventral*category) & 0.495 & 7 & 0.071 & & \\
\hline region $*$ hemisphere $*$ dorsal_ventral $*$ category & 0.082 & 3 & 0.027 & 2.434 & .093 \\
\hline
\end{tabular}




\begin{tabular}{|c|c|c|c|c|c|}
\hline Error(region*hemisphere*dorsal_ventral*category) & 0.235 & 21 & 0.011 & & \\
\hline left_rightHemi * category & 1.152 & 1 & 1.152 & 6.228 & 0.041 \\
\hline Error(left_rightHemi*category) & 1.295 & 7 & 0.185 & & \\
\hline region * left_rightHemi * category & 0.087 & 3 & 0.029 & 1.369 & 0.28 \\
\hline Error(region*left_rightHemi*category) & 0.447 & 21 & 0.021 & & \\
\hline hemisphere * left_rightHemi * category & 0.674 & 1 & 0.674 & 13.65 & 0.008 \\
\hline Error(hemisphere*left_rightHemi*category) & 0.346 & 7 & 0.049 & & \\
\hline region $*$ hemisphere $*$ left_rightHemi * category & 0.241 & 3 & 0.08 & 2.376 & 0.099 \\
\hline Error(region*hemisphere*left_rightHemi*category) & 0.71 & 21 & 0.034 & & \\
\hline dorsal_ventral * left_rightHemi * category & 1.324 & 1 & 1.324 & 15.596 & 0.006 \\
\hline Error(dorsal_ventral*left_rightHemi*category) & 0.594 & 7 & 0.085 & & \\
\hline region * dorsal_ventral * left_rightHemi * category & 0.152 & 3 & 0.051 & 4.073 & 0.02 \\
\hline Error(region*dorsal_ventral*left_rightHemi*category) & 0.262 & 21 & 0.012 & & \\
\hline hemisphere * dorsal_ventral * left_rightHemi $*$ category & 0.094 & 1 & 0.094 & 2.465 & 0.16 \\
\hline $\begin{array}{l}\text { Error(hemisphere*dorsal_ventral*left_rightHemi*category) } \\
\text { region }{ }^{*} \text { hemisphere }{ }^{*} \text { dorsal_ventral }{ }^{*} \text { left_rightHemi }{ }^{*}\end{array}$ & 0.266 & 7 & 0.038 & & \\
\hline category & 0.004 & 3 & 0.001 & 0.033 & 0.992 \\
\hline $\begin{array}{l}\text { Error(region*hemisphere*dorsal_ventral*left_rightHemi*ca } \\
\text { tegory) }\end{array}$ & 0.814 & 21 & 0.039 & & \\
\hline upper_lowerVF * category & 0.145 & 1 & 0.145 & 1.2 & 0.31 \\
\hline Error(upper_lowerVF*category) & 0.844 & 7 & 0.121 & & \\
\hline region * upper_lowerVF * category & 0.092 & 3 & 0.031 & 1.13 & 0.36 \\
\hline Error(region*upper_lowerVF*category) & 0.568 & 21 & 0.027 & & \\
\hline hemisphere * upper_lowerVF * category & 0.513 & 1 & 0.513 & 13.893 & 0.007 \\
\hline Error(hemisphere*upper_lowerVF*category) & 0.259 & 7 & 0.037 & & \\
\hline region * hemisphere * upper_lowerVF * category & 0.062 & 3 & 0.021 & 0.655 & 0.589 \\
\hline Error(region*hemisphere*upper_lowerVF*category) & 0.666 & 21 & 0.032 & & \\
\hline dorsal_ventral * upper_lowerVF * category & 0.689 & 1 & 0.689 & 7.25 & 0.031 \\
\hline Error(dorsal_ventral*upper_lowerVF*category) & 0.665 & 7 & 0.095 & & \\
\hline region * dorsal_ventral * upper_lowerVF * category & 0.024 & 3 & 0.008 & 0.582 & 0.633 \\
\hline Error(region*dorsal_ventral*upper_lowerVF*category) & 0.289 & 21 & 0.014 & & \\
\hline $\begin{array}{l}\text { hemisphere * dorsal_ventral * upper_lowerVF * category } \\
\text { Error(hemisphere*dorsal_ventral*upper_lowerVF*category }\end{array}$ & 0.33 & 1 & 0.33 & 1.746 & 0.228 \\
\hline region $*$ hemisphere $*$ dorsal_ventral $*$ upper_lowerVF $*$ & 1.321 & 7 & 0.189 & & \\
\hline $\begin{array}{l}\text { category } \\
\text { Error(region*hemisphere*dorsal_ventral*upper_lowerVF*c } \\
\text { ategory) }\end{array}$ & 0.234 & 21 & 0.078 & 1.739 & 0.19 \\
\hline left_rightHemi $*$ upper_lowerVF * category & 1.018 & 1 & 1.018 & 2.327 & 0.171 \\
\hline Error(left_rightHemi*upper_lowerVF*category) & 3.063 & 7 & 0.438 & & \\
\hline region * left_rightHemi * upper_lowerVF * category & 0.168 & 3 & 0.056 & 1.835 & 0.172 \\
\hline Error(region*left_rightHemi*upper_lowerVF*category) & 0.641 & 21 & 0.031 & & \\
\hline $\begin{array}{l}\text { hemisphere * left_rightHemi * upper_lowerVF * category } \\
\text { Error(hemisphere*left_rightHemi*upper_lowerVF*category }\end{array}$ & 0.033 & 1 & 0.033 & 0.606 & 0.462 \\
\hline region * hemisphere * left_rightHemi * upper_lowerVF * & 0.38 & 7 & 0.054 & & \\
\hline category & 0.054 & 3 & 0.018 & 0.836 & 0.489 \\
\hline Error(region*hemisphere*left_rightHemi*upper_lowerVF*c & 0.451 & 21 & 0.021 & & \\
\hline
\end{tabular}


ategory)

\begin{tabular}{|c|c|c|c|c|c|}
\hline $\begin{array}{l}\text { dorsal_ventral }{ }^{*} \text { left_rightHemi * upper_lowerVF }{ }^{*} \text { category } \\
\text { Error(dorsal_ventral*left_rightHemi*upper_lowerVF*catego }\end{array}$ & $6.90 \mathrm{E}-05$ & 1 & $6.90 \mathrm{E}-05$ & 0.001 & 0.981 \\
\hline $\begin{array}{l}\text { ry) } \\
\text { region } * \text { dorsal ventral } * \text { left rightHemi } * \text { upper lowerVF } *\end{array}$ & 0.801 & 7 & 0.114 & & \\
\hline $\begin{array}{l}\text { category } \\
\text { Error(region*dorsal_ventral*left_rightHemi*upper_lowerVF }\end{array}$ & 0.1 & 3 & 0.033 & 2.162 & 0.123 \\
\hline $\begin{array}{l}\text { *category) } \\
\text { hemisphere } * \text { dorsal ventral } * \text { left rightHemi } *\end{array}$ & 0.324 & 21 & 0.015 & & \\
\hline $\begin{array}{l}\text { upper_lowerVF * category } \\
\text { Error(hemisphere*dorsal ventral*left rightHemi*upper lo }\end{array}$ & 0.627 & 1 & 0.627 & 15.707 & 0.005 \\
\hline $\begin{array}{l}\text { werVF* category) } \\
\text { region } * \text { hemisphere } * \text { dorsal_ventral } * \text { left_rightHemi } *\end{array}$ & 0.279 & 7 & 0.04 & & \\
\hline $\begin{array}{l}\text { upper_lowerVF * category } \\
\text { Error(region*hemisphere*dorsal_ventral*left_rightHemi*up }\end{array}$ & 0.025 & 3 & 0.008 & 0.795 & 0.51 \\
\hline per_lowerVF*'category) & 0.223 & 21 & 0.011 & & \\
\hline
\end{tabular}


Appendix 2: Standard deviation of correlation matrix shown in Figure 8 
a. Experimental task
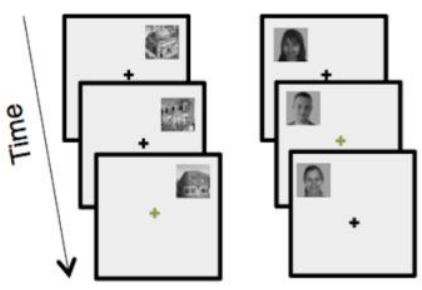

b. Stimulus locations c. Localizer scans

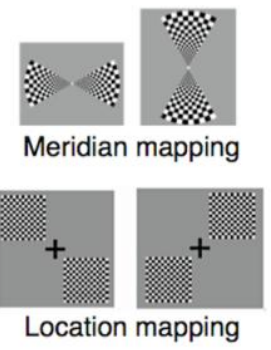

बृत्

(P)

Face/House 

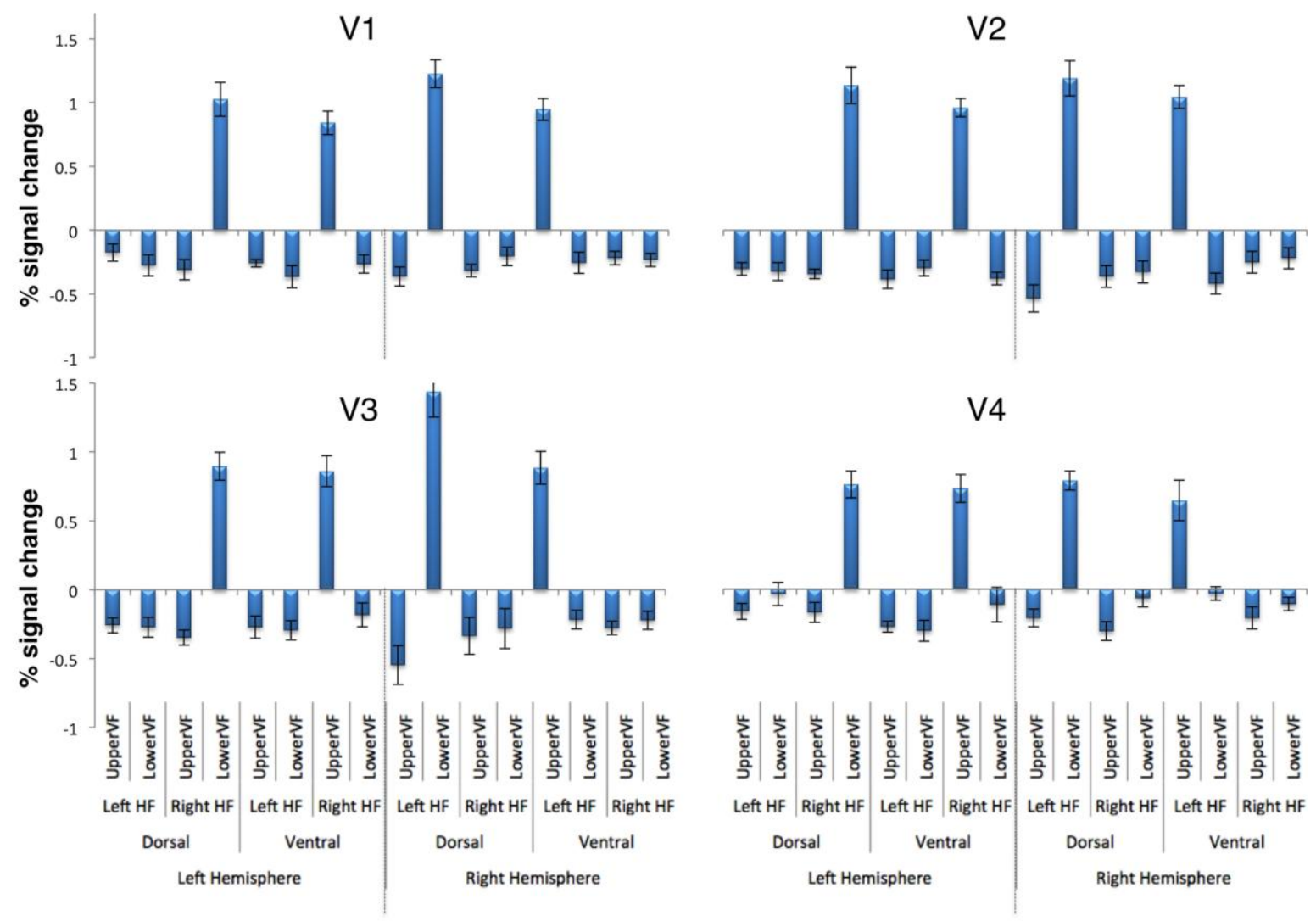

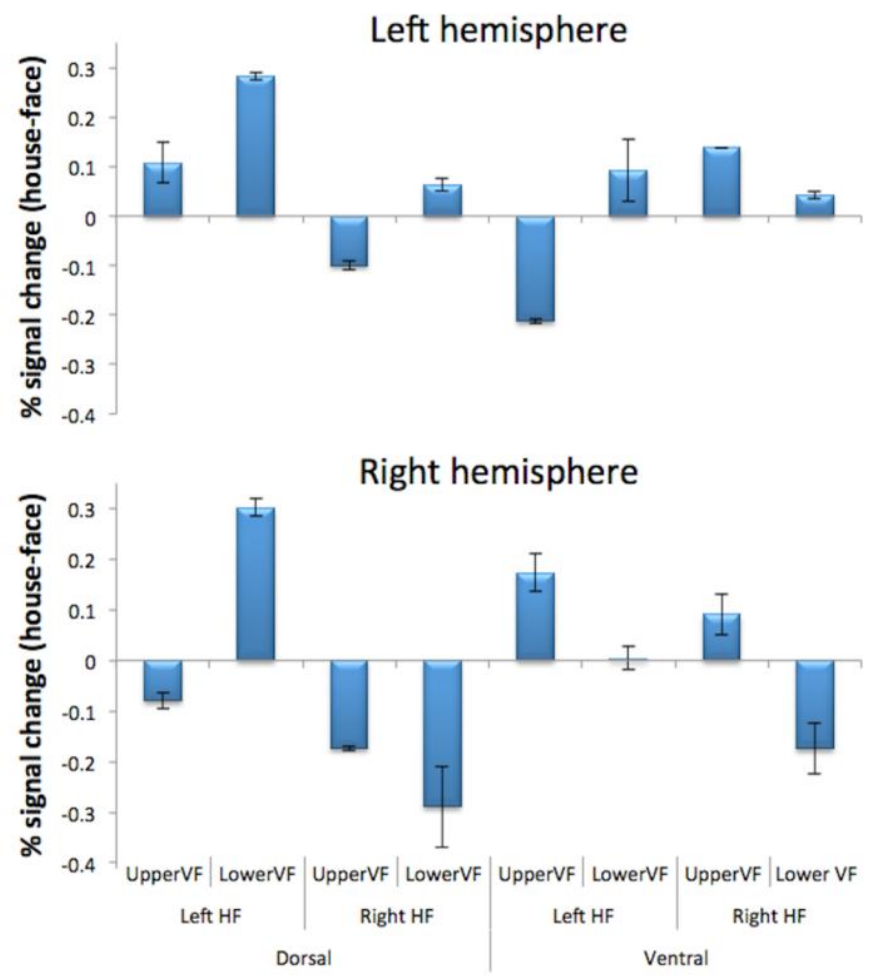


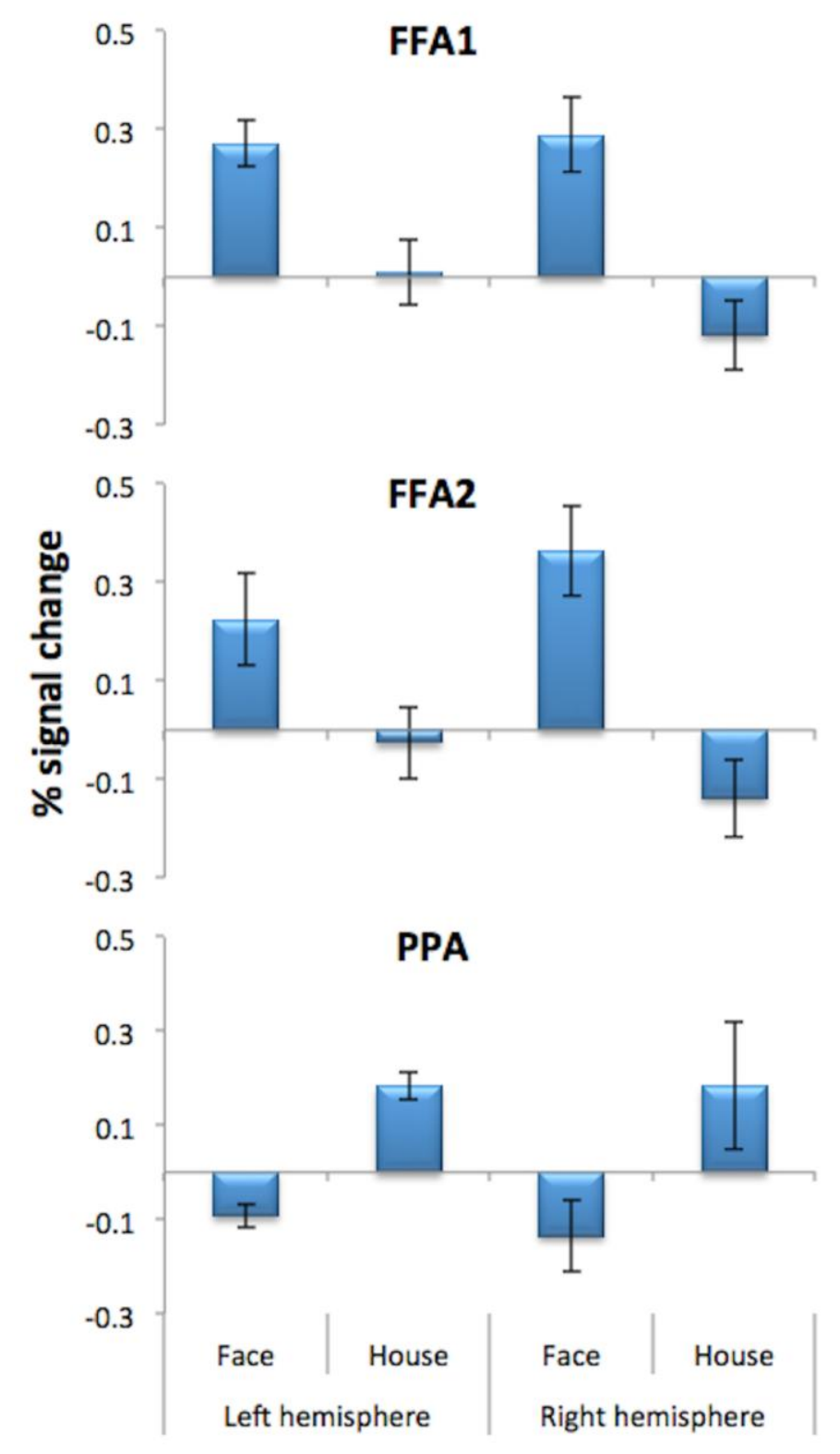



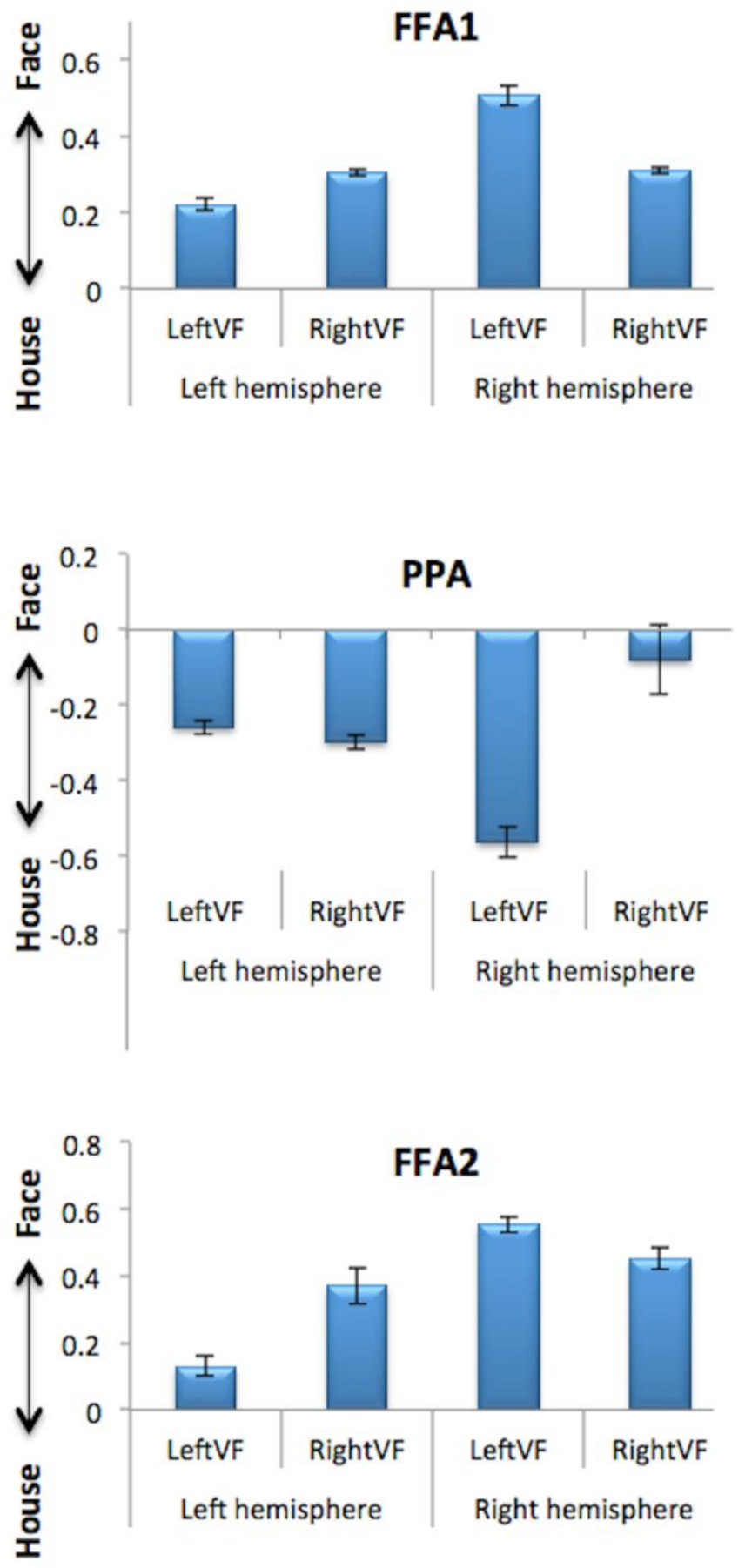

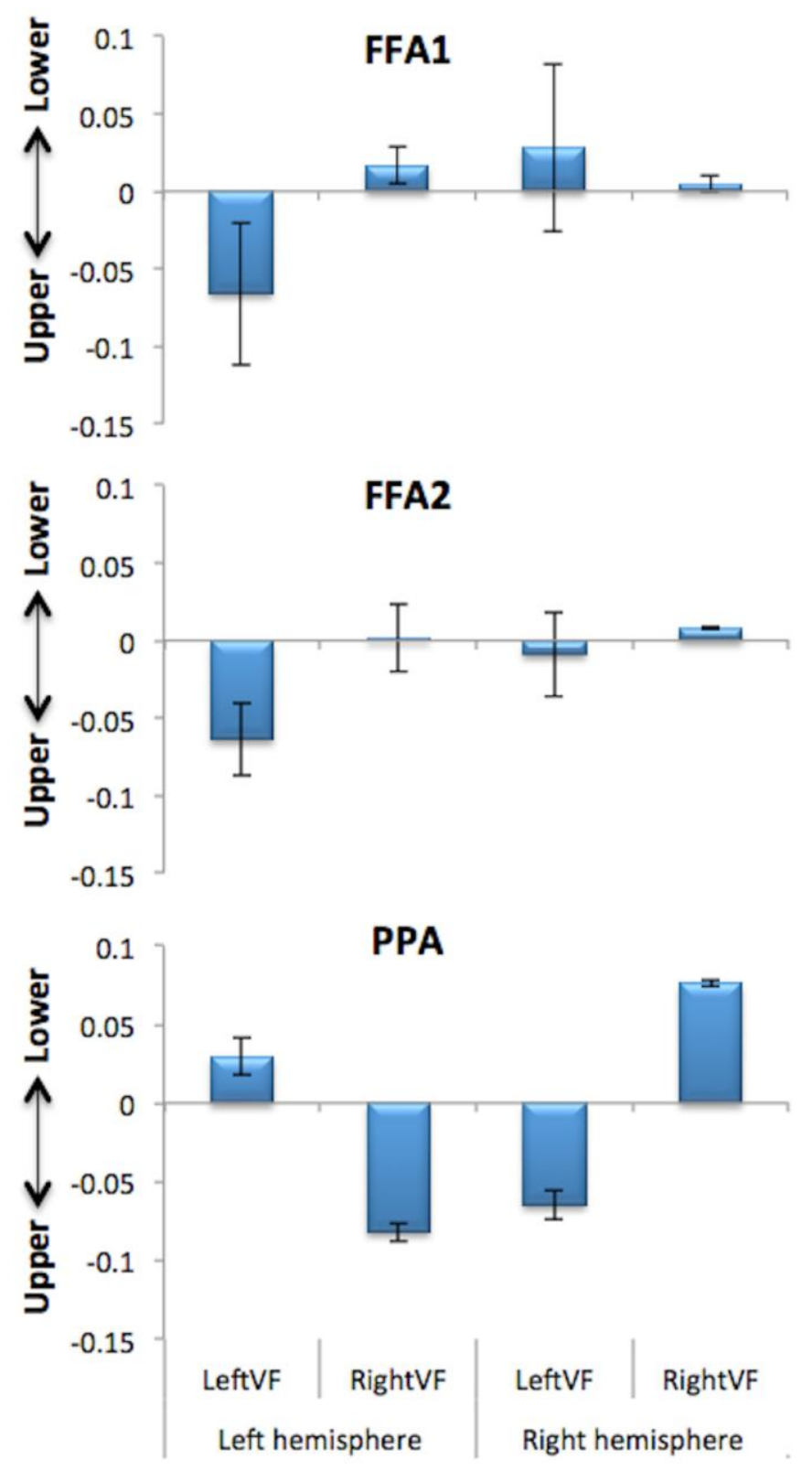

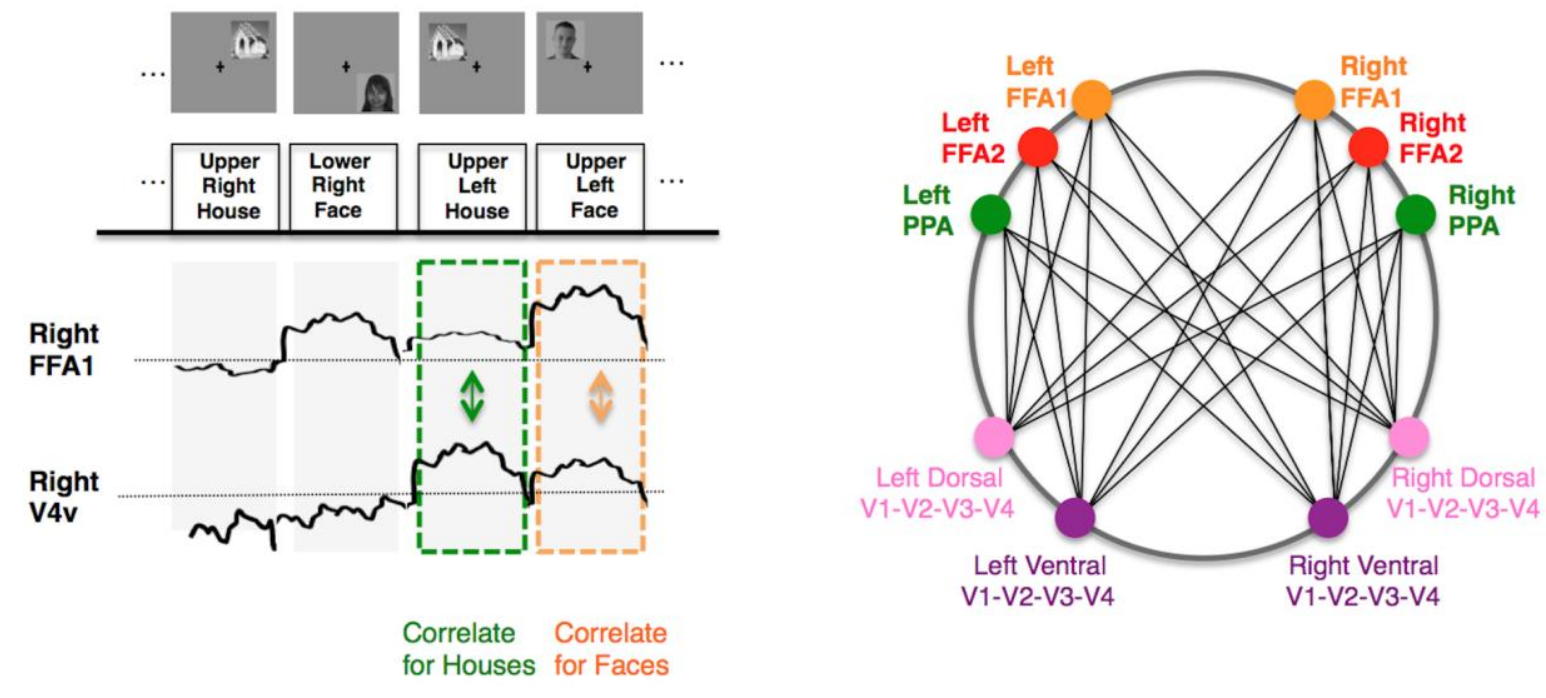


\begin{tabular}{|c|c|c|c|c|c|c|c|c|c|c|c|c|c|c|c|c|}
\hline FACE & lh_v1d & Ih_v2d & Ih_v3d & h_v4d & Ih_v1v & lh_v2v & Ih_v3v & lh_v4v r & h_v1d & rh_v2d r & rh_v3dr & rh_v4d r & hh_v1v & rh_v2v & rh_v3v & rh_v4v \\
\hline LFFA1 & 0.43 & 0.42 & 0.34 & 0.41 & 0.54 & 0.61 & 0.58 & 0.62 & 0.21 & 0.14 & 0.05 & 0.18 & 0.34 & 0.30 & 0.30 & 0. \\
\hline LFFA2 & 0.30 & 0.25 & 0.19 & 0.27 & 0.32 & 0.34 & 0.29 & 0.34 & 0.18 & 0.04 & 0.06 & 0.07 & 0.15 & 0.19 & 0.28 & \\
\hline RFFA1 & 0.14 & 0.07 & 0.09 & 0.13 & 0.35 & 0.38 & 0.31 & 0.33 & 0.40 & 0.31 & 0.24 & 0.33 & 0.53 & 0.46 & 0.47 & \\
\hline RFFA2 & 0.17 & 0.14 & 0.08 & 0.12 & 0.37 & 0.38 & 0.32 & 0.35 & 0.25 & 0.20 & 0.18 & 0.15 & 0.42 & 0.34 & 0.38 & 0 \\
\hline LPPA & 0.19 & 0.17 & 0.08 & 0.16 & 0.31 & 0.27 & 0.42 & 0.48 & -0.15 & -0.18 & -0.17 & -0.07 & 0.15 & 0.17 & 0.21 & 0.2 \\
\hline RPPA & 0.17 & 0.12 & 0.07 & 0.12 & 0.21 & 0.16 & 0.23 & 0.24 & 0.17 & 0.08 & 0.07 & 0.05 & 0.33 & 0.30 & 0.41 & 1] \\
\hline
\end{tabular}

\begin{tabular}{|c|c|c|c|c|c|c|c|c|c|c|c|c|c|c|c|c|}
\hline HOUSE & v1d & h_v2d & h & Ih_v4d & h_v1v & Ih_v2v & Ih_v3v & Ih_v4v & rh_v1d & rh_v2d & h_v3d & rh_v4d & rh_v1v & rh_v2v & rh_v3v & rh_v $4 v$ \\
\hline LFFA1 & 0.28 & 0.28 & 0.30 & 0.28 & 0.42 & 0.46 & 0.46 & 0.49 & 0.18 & 0.16 & 0.12 & 0.17 & 0.19 & 0.15 & 0.22 & 0. \\
\hline LFFA2 & 0.13 & 0.13 & 0.12 & 0.10 & 0.12 & 0.16 & 0.17 & 0.18 & 0.23 & 0.09 & 0.13 & 0.13 & 0.06 & 0.04 & 0.21 & \\
\hline RFFA1 & 0.15 & 0.07 & 0.12 & 0.14 & 0.24 & 0.26 & 0.22 & 0.22 & 0.30 & 0.18 & 0.20 & 0.25 & 0.29 & 0.28 & 0.38 & \\
\hline RFFA2 & -0.09 & -0.08 & -0.07 & -0.05 & 0.10 & 0.12 & 0.08 & 0.07 & 0.17 & 0.16 & 0.12 & 0.19 & 0.16 & 0.18 & 0.23 & 0.22 \\
\hline LPPA & 0.31 & 0.27 & 0.26 & 0.23 & 0.32 & 0.36 & 0.38 & 0.45 & 0.25 & 0.13 & 0.19 & 0.17 & 0.24 & 0.26 & 0.21 & 0.23 \\
\hline RPPA & 0.14 & 0.10 & 0.09 & 0.15 & 0.24 & 0.27 & 0.36 & 0.30 & 0.37 & 0.33 & 0.33 & 0.34 & 0.44 & 0.47 & 0.48 & 0.4 \\
\hline
\end{tabular}

